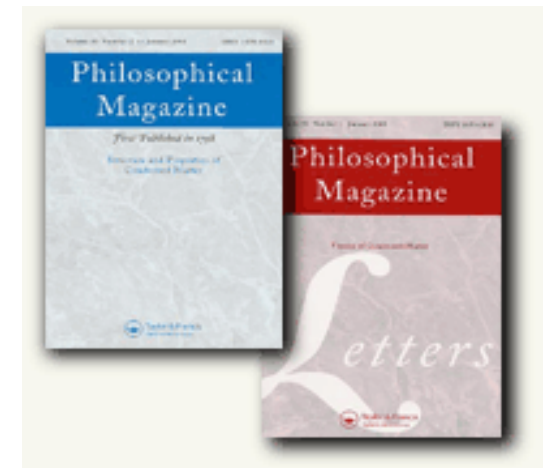

\title{
Iron chromium potential to model high-chromium ferritic alloys
}

\begin{tabular}{|r|l|}
\hline Journal: & Philosophical Magazine \& Philosophical Magazine Letters \\
\hline Manuscript ID: & TPHM-10-Sep-0405.R2 \\
\hline Journal Selection: & Philosophical Magazine \\
\hline Date Submitted by the \\
Author: & 02-Dec-2010 \\
\hline Complete List of Authors: & $\begin{array}{l}\text { Bonny, Giovanni; SCK-CEN, Nuclear Materials Science Institute } \\
\text { Pasianot, Roberto; CNEA } \\
\text { Terentyev, Dmitry; SCK-CEN } \\
\text { Malerba, Lorenzo; SCK-CEN }\end{array}$ \\
\hline Keywords: & $\begin{array}{l}\text { interatomic potential, atomistic simulation, thermodynamics, } \\
\text { dislocations }\end{array}$ \\
\hline Keywords (user supplied): & iron chromium alloys \\
\hline \multicolumn{2}{|c}{} \\
\hline
\end{tabular}

\section{SCHOLARONE \\ Manuscripts}


${ }^{1}$ SCK $•$ CEN, Nuclear Material Science Institute, Boeretang 200, B-2400, Mol, Belgium

${ }^{2}$ CAC-CNEA, Depto. de Materiales, Avda. Gral. Paz 1499, 1650 San Martín, Pcia. Buenos Aires, Argentina

${ }^{3}$ CONICET, Avda. Rivadavia 1917, 1033 Buenos Aires, Argentina

${ }^{4}$ UNSAM/CNEA, Intituto Sabato, Avda. Gral. Paz 1499, 1650 San Martín, Pcia. Buenos Aires, Argentina

\begin{abstract}
In this paper we present an $\mathrm{Fe}-\mathrm{Cr}$ interatomic potential to model high-Cr ferritic steels. The potential is fitted to thermodynamic and point-defect properties obtained from density functional theory (DFT) calculations and experiments. The here developed potential is also benchmarked against other potentials available in literature. It shows particularly good agreement with the DFT obtained mixing enthalpy of the random alloy, the formation energy of intermetallics and experimental excess vibrational entropy and phase diagram. Also DFT calculated point-defect properties, both interstitial and substitutional, are well reproduced as is the screw dislocation core structure. As a first validation of the potential we study the precipitation hardening of $\mathrm{Fe}-\mathrm{Cr}$ alloys by means of static simulations of the interaction between $\mathrm{Cr}$ precipitates and screw dislocations. It is concluded that the description of the dislocation core modification near a precipitate might have a significant influence on the interaction mechanisms observed in dynamic simulations.
\end{abstract}

Keywords: interatomic potential; atomistic modelling; iron chromium alloys; thermodynamics; dislocations

PACS: 81.30.-t; 81.40.Cd; 61.72.J-; 61.72.Lk

\title{
1. Introduction
}

High-chromium ferritic-martensitic steels ( 9-12 at.\% Cr) are the materials of choice for high temperature applications in aggressive environments (e.g. corrosion and/or irradiation). As a 
consequence, they are the commonly proposed structural materials for advanced nuclear reactors. This choice is supported by their superior thermal, corrosion and radiation resistance as compared to austenitic steels. A first approximation to describe such steels in a modelling framework is the $\mathrm{Fe}-\mathrm{Cr}$ binary alloy.

The addition of chromium to iron influences significantly the response to irradiation. It has been shown that the swelling in $\mathrm{Fe}-\mathrm{Cr}$ is about one order of magnitude lower than in pure Fe for the same dose $[1 ; 2 ; 3 ; 4 ; 5]$. A remarkable effect of $\mathrm{Cr}$ is also reported in the shift of the ductile-to-brittle transition temperature (DBTT) in irradiated ferritic-martensitic steels. This shift is found to reach a minimum around 9 at.\% $\mathrm{Cr}[6 ; 7]$, in a range of irradiation temperatures from 300 to $410^{\circ} \mathrm{C}$ and for doses from 7 to $36 \mathrm{dpa}$. This result is in fact an important reason for choosing $\mathrm{Cr}$ concentrations around 9 at.\% in most steels proposed for nuclear applications [7].

Even in the absence of irradiation, the $\mathrm{Fe}-\mathrm{Cr}$ system exhibits a number of peculiarities. Density functional theory (DFT) calculations have shown that the mixing enthalpy exhibits a change of sign $[8 ; 9 ; 10 ; 11 ; 12 ; 13 ; 14]$, which is negative below a critical concentration of $\sim 10$ at. $\% \mathrm{Cr}$ and positive above it. A negative mixing enthalpy indicates solubility and a tendency to partial ordering, while a positive value indicates that $\mathrm{Cr}$ atoms cluster into a separate Cr-rich phase ( $\alpha^{\prime}$ phase). These results are compatible with experimental observations that show an inversion of sign of the short-range order (SRO) parameter from negative to positive, with the zero crossing occurring at about 10 at.\% $\mathrm{Cr}[15 ; 16 ; 17]$. However, this behaviour is not reflected in the standard $\mathrm{Fe}-\mathrm{Cr}$ equilibrium phase diagram [18], whose revision was therefore recently proposed [19] by shifting the concentration of the Fe-rich phase boundary of the meta-stable (when ignoring the sigma phase) miscibility gap to a larger $\mathrm{Cr}$ value ( $\sim 8$ at.\% $\mathrm{Cr}$ ), even at low temperature.

In order to better understand the behaviour of $\mathrm{Fe}-\mathrm{Cr}$ alloys under irradiation, a description at the atomic level is necessary. To address problems of technological interest (e.g. plastic flow behaviour and evolution of the nano- and microstructure), however, descriptions at larger time and space scales are necessary. Both small and large-scale atomistic simulations essentially depend on the interatomic potentials employed, which therefore must strike a balance between the conflicting needs of computational speed and reliability. Thus, an interatomic potential describing the $\mathrm{Fe}-\mathrm{Cr}$ alloy reasonably well in the temperature range of interest is needed.

* Author for correspondence. Email: gbonny@ sckcen.be; Tel.: +32-14-333197; fax: +32-14-321216. 


\section{Formalism and Fitting Methodology}

It has been shown that both the $2 \mathrm{BM}$ and the $\mathrm{CDM}$ formalisms are adequate models to describe Fe-Cr's complex mixing enthalpy without accounting for magnetism explicitly [23]. Here we choose the $2 \mathrm{BM}$ formalism, where the total energy is given as in the EAM with an extra embedding term, $F^{s}$,

$$
E=\frac{1}{2} \sum_{i, j(i \neq j)} V_{t_{i} t_{j}}\left(r_{i j}\right)+\sum_{i} F_{t_{i}}^{\mathrm{d}}\left(\rho_{i}^{\mathrm{d}}\right)+\sum_{i} F_{t_{i}}^{\mathrm{s}}\left(\rho_{i}^{\mathrm{s}}\right),
$$


The additional embedding term on the right-hand side is assumed to stem from the $s$-band electrons, while the standard embedding, $F^{\mathrm{d}}$, is thought of as resulting from the $d$-band electrons [20]; $t_{i}$ denotes the atom type (in our case Fe or $\mathrm{Cr}$ ) on site $i$. The electron densities, $\rho^{\mathrm{d}}$ and $\rho^{s}$, for the $d$ - and $s$-band, respectively, are calculated as,

$$
\rho_{i}^{\lambda}=\sum_{j(j \neq i)} \varphi_{t_{i} t_{j}}^{\lambda}\left(r_{i j}\right)
$$

Here $\varphi^{\mathrm{d}}$ and $\varphi^{\mathrm{s}}$ are the $d$ - and $s$-density functions, respectively, which comply with the relations,

$$
\left\{\begin{array}{l}
\varphi_{A A}^{d}=\varphi_{B A}^{d}=\varphi_{A}^{d} \\
\varphi_{B B}^{d}=\varphi_{A B}^{d}=\varphi_{B}^{d} \\
\varphi_{A A}^{s}=\varphi_{B B}^{s}=0 \\
\varphi_{A B}^{s}=\varphi_{B A}^{s}
\end{array}\right.
$$

This choice is in line with [20] so that the $d$-density reduces to the EAM density, and the $s$ density samples the local concentration [23]. In this way the $s$-embedding terms are only relevant for the alloy, and do not contribute to the pure elements' energy, which keeps the standard EAM expression.

Thus, for the Fe-Cr system and within the 2BM, ten functions need to be determined: $V_{\mathrm{FeFe}}, F_{\mathrm{Fe}}^{\mathrm{d}}, \varphi_{\mathrm{Fe}}^{\mathrm{d}}, V_{\mathrm{CrCr}}, F_{\mathrm{Cr}}^{\mathrm{d}}, \varphi_{\mathrm{Cr}}^{\mathrm{d}}, V_{\mathrm{FeCr}}, F_{\mathrm{Fe}}^{\mathrm{s}}, F_{\mathrm{Cr}}^{\mathrm{s}}$ and $\varphi_{\mathrm{FeCr}}^{\mathrm{s}}$. The first three functions determine the $\mathrm{Fe}$ potential, the second three the $\mathrm{Cr}$ potential and the last four the mixed $\mathrm{Fe}-\mathrm{Cr}$ interactions. For pure Fe, we chose the potential developed by Mendelev et al. [24], while the pure $\mathrm{Cr}$ potential and the mixed $\mathrm{Fe}-\mathrm{Cr}$ interactions were fitted as is described further on. As is also better detailed further on, the main reason to refit the potential for pure $\mathrm{Cr}$ is to provide a better description of dislocation properties in this metal. Generally, the fitting of an interatomic potential to material properties is a problem of matching data obtained from a trial function to a given data set. This problem can be cast into one of minimizing the overall squared deviation, so-called objective function (OF), between the calculated properties and the associated reference data, possibly also imposing constraints. Within our methodology, those properties become linear in the fitting parameters, reducing the scheme to a quadratic programming problem [25] which is exactly solvable; more details are given below. 
The pair interaction $V$ is parameterized by the cubic spline expansion,

$$
V(r)=\sum_{k=1}^{N} a_{k}\left(r_{k}-r\right)^{3} \Theta\left(r_{k}-r\right)
$$

where $N$ denotes the number of knots, $a_{k}$ are the fitting parameters and $\Theta$ the Heaviside unit step function. As explained below, the embedding term $F$ is only available in numerical format.

The essential material properties considered in the fit for pure $\mathrm{Cr}$ (anti-ferromagnetic phase unless stated otherwise) are the correct stability of different crystallographic structures, the cohesive energy $E_{\mathrm{coh}}$, the equilibrium lattice constant $a_{0}$, the elastic constants (for cubic lattice structures) $C_{11}, C_{12}, C_{44}$ [26] (paramagnetic phase, see Section 3), and the vacancy formation energy, $E_{f}^{\vee}$. In addition to these, we also included in the fit the formation energy of self-interstitial configurations (dumbbells oriented along the $\langle 100\rangle,\langle 110\rangle$ and $\langle 111\rangle$ 
directions), the vacancy migration energy, $E_{\mathrm{m}}^{\vee}$, the $\{110\}\langle 111\rangle$ gamma surface cut, $\gamma$, and the $1 / 2\langle 111\rangle$ screw-dislocation core structure. In particular, the gamma surface cut ( 10 points) was fitted through the OF, only using the effective pair potential (i.e. neglecting higher order many-body contributions) [27]. The lattice parameter (zero pressure condition), unrelaxed vacancy formation energy, $E_{f}^{\vee}$, and the three elastic constants were fitted through equality constraints. The bcc lattice stability against fcc $\left(E_{\mathrm{coh}}(\mathrm{bcc})-E_{\mathrm{coh}}(\mathrm{fcc})>0\right)$ and the Duesbery \& Vitek condition for a compact $1 / 2\langle 111\rangle$ screw dislocation core $(\chi(b / 3)-2 \chi(b / 6)>0$ [28], with $b$ the length of the burgers vector) were fitted through inequality constraints.

Given the effective pair potential, the embedding function is computed by subtraction from Rose's equation [29], which describes the energy per atom $E(\tilde{a})$ for the lattice under uniform expansion or contraction. The expression as a function of the nearest neighbour distance, $r^{1 \mathrm{nn}}$, is given as,

$$
E(\tilde{a})=-E_{\mathrm{coh}}(1+\tilde{a}) \exp (-\tilde{a}) .
$$

Here $\tilde{a}=\alpha\left(r^{\operatorname{lnn}} / r_{0}^{\ln }-1\right)$ and $\alpha^{2}=9 \Omega B / E_{\text {coh }}$, where $\Omega$ is the atomic volume and $B$ is the bulk modulus. This embedding function, however, is not the one entering the potential. In order to ensure a smooth behaviour and particularly, to constrain the function curvature to remain positive throughout and decreasing at high density, the former embedding is re-fitted on a discrete number of points $(\sim 100)$ taken as reference data. The fit is performed in a minimum square sense and by a cubic spline expansion (also fulfilling $\left.F^{\prime}(0)=0\right)$. It is the latter expansion that constitutes the embedding function. The price paid for a smooth behaviour is a violation of Rose's equation; however, in the applications of interest the lattice will never be subjected to the large, uniform contractions and dilations that are probed by Rose's equation. Finally, the last fitting step consisted in accommodating manually (i.e. by trial and error) the short distance region to fit the DFT obtained values for self-interstitial formation energies.

\subsection{Alloy Potential}

Given the EAM potentials for the pure elements and prior to fitting $V_{\mathrm{FeCr}}, F_{\mathrm{Fe}}^{\mathrm{s}}$ and $F_{\mathrm{Cr}}^{\mathrm{s}}$, we define a reasonable form for the $s$-electron density function $\varphi_{\mathrm{FeCr}}^{\mathrm{s}}$. In line with [20], the square of a $4 s$-type Slater function is chosen, 


$$
\varphi_{\mathrm{FeCr}}^{\mathrm{s}}(r)=K r^{6} \exp (-2 \zeta r) g_{\text {cut }}(r)
$$

Here $K=20.34075425$ is a normalisation constant, $\zeta=2.5001$ and $g_{\text {cut }}$ is a cut-off function to provide a smooth cut-off between fifth and sixth nearest neighbour distance,

$$
g_{\text {cut }}(r)=\left\{\begin{array}{lc}
1 & r \leq r_{c}^{i} \\
\frac{1}{2}\left\{1-\sin \left[\frac{\pi}{2} \frac{\left(r-r_{m}\right)}{d}\right]\right\}, r_{c}^{i}<r \leq r_{c}^{f}, \\
0, r_{c}^{f}<r
\end{array}\right.
$$

with $r_{c}^{i}=5.1 \AA$ the inner cut-off, $r_{c}^{f}=5.3 \AA$ the outer cut-off, $r_{m}=\left(r_{c}^{i}+r_{c}^{f}\right) / 2$ and $d=\left(r_{c}^{f}-r_{c}^{i}\right) / 2$. The mixed pair interaction $V_{\mathrm{FeCr}}$ is parameterized by a cubic spline expansion as in Equation (6) while the $s$-embedding functions $F^{s}$ are parameterized by an extended second moment expression similar to [20; 30],

$$
F^{s}(\rho)=A_{1} \sqrt{\rho}+A_{2} \rho^{2},
$$

with $A_{1}$ and $A_{2}$ fitting parameters. The square root proved to be essential in reproducing the correct trends in $\mathrm{Cr}-\mathrm{Cr}$ and $\mathrm{Cr}$-vacancy interactions. In fact, former attempts using spline expansions as in [23] were met with failure. In this work we normalized the (maximum) $s$ density in such a way that unity corresponds to a single $\mathrm{Cr}$ hosted in the $\mathrm{Fe}$ matrix.

The mixed pair interaction, as well as both $s$-embedding functions, were fitted simultaneously to all properties of interest. The mixing enthalpy was fitted in the whole concentration range ( $\sim 50$ points) using a variance expansion as described in [23], while at each concentration a zero pressure condition was imposed to obtain a lattice parameter as close as possible to the linear interpolation between the equilibrium lattice parameters of the pure elements. In addition, the $\mathrm{Cr}$ - $\mathrm{Cr}$ and $\mathrm{Cr}$-vacancy interaction energies up to second nearest neighbour were fitted, as well as the Cr-vacancy migration energy in Fe, using DFT reference values as guides [12]. All the mentioned properties were fitted using appropriate weights to obtain reasonable values. In addition to the expansion coefficients of the $s$-embedding functions and the mixed pair potential, there remains one degree of freedom that expresses the relative weight between the $d$-densities of $\mathrm{Fe}$ and $\mathrm{Cr}, \rho_{\mathrm{Fe}} / \rho_{\mathrm{Cr}}$. By manually changing this 
fraction and consequently solving the quadratic programming problem, we fitted the excess vibrational entropy, computed in the harmonic approximation [31], to a value as close as possible to the experimental one, without losing the above properties and without stabilizing unphysical intermetallic compounds. Finally, the potential was manually adjusted in the short distance region to reproduce the DFT-obtained binding energy of the mixed $\langle 110\rangle \mathrm{Fe}-\mathrm{Cr}$ dumbbell. A description of the full $\mathrm{Fe}-\mathrm{Cr}$ potential is given in Appendix A.

\section{Properties of the Iron and Chromium Potentials}

From the EAM-type potentials available in the literature to describe pure Fe that are capable of reproducing the $\langle 110\rangle$ self-interstitial configuration as the most stable, we considered those from Refs. [24], [32] and [33], finally selecting "potential 2" developed by Mendelev et al. [24], which has been widely used and tested. In [34] it has been demonstrated that the potentials from [24] and [32] succeed best in reproducing the properties of iron that are of interest for radiation damage studies. However, of all, our choice is the only one for which a clear proof exists of being able to consistently describe stable glide of a screw dislocation in a $\{110\}$ plane [35].

Among the potentials for $\mathrm{Cr}$, we considered those from Refs. [36] and [20]. The latter, henceforth $\mathrm{O} 05$, was used in combination with the Fe potential by Ackland et al. [32] to produce the OLS and CAR potentials. The basic properties of our potential are summarized and compared to O05 and DFT data in Table 1. There, $B=\left(C_{11}+2 C_{12}\right) / 3$ is the bulk modulus, $C^{\prime \prime}=\left(C_{11}-C_{12}\right) / 2$ is the tetragonal shear and $C_{44}$ is the shear modulus [26]. Clearly, the experimental elastic properties are well reproduced by both potentials. Note, however, that at zero Kelvin $\mathrm{Cr}$ is anti-ferromagnetic and has a negative Cauchy pressure [37], which cannot be reproduced within a standard central force framework [38; 39; 40]. Above its Néel temperature $(310 \mathrm{~K})$, however, $\mathrm{Cr}$ is paramagnetic with a positive Cauchy pressure [41]. Since we focus on technological applications above room temperatures, our potential was fitted to the elastic constants of paramagnetic $\mathrm{Cr}$, linearly extrapolated down to zero Kelvin (the values given in Table 1, see e.g. [36]). Furthermore, our potential also closely fits $a_{0}, E_{\mathrm{f}}^{\vee}$, $E_{\mathrm{coh}}$ and $E_{\mathrm{m}}^{\vee}$, obtaining improved values for the latter two. The relative stability between the different self interstitial configurations is not equally well reproduced as by $\mathrm{O} 05$, but the important trends given by DFT are reproduced, i.e., the $\langle 110\rangle$ and $\langle 111\rangle$ configurations are almost degenerate and more stable than the $\langle 100\rangle$ configuration. Finally, our potential is closer 
to DFT data regarding the energy difference between the body-centred cubic (bcc) and facecentred cubic (fcc) phase, thus providing enhanced stability of the bcc phase at high temperature. In summary, from the values presented in Table 1 we can conclude that the basic properties of $\mathrm{Cr}$ are equally well reproduced by both potentials.

\section{Instert table 1 around here}

The O05 potential, however, does not reproduce the correct structure for a $1 / 2\langle 111\rangle$ screw-dislocation core, which is the main reason to refit the $\mathrm{Cr}$ potential. This is depicted in Figure 1 via differential displacement maps [42]. Clearly 005 shows a three-fold symmetry core, opposite to the compact one, as predicted by DFT [43] and our potential.

\section{Insert figure 1 around here}

\section{Thermodynamic Properties}

Prior to presenting the phase diagrams resulting from the different potentials, we compare and discuss statically calculated properties that influence the $\mathrm{Fe}-\mathrm{Cr}$ phase diagram. In Figure 2 the mixing enthalpy calculated with the three potentials is compared to DFT data $[8 ; 12 ; 44]$. For the mixing enthalpy resulting from the potentials 50 compositions in the complete concentration range were sampled. For each composition the solutes were randomly distributed in cubic bcc crystals containing 2000 atoms and relaxed at constant pressure using a conjugate gradient method. Although different DFT techniques give different values for the mixing enthalpy, the DFT curves show the same behaviour, i.e., a change of sign in the mixing enthalpy, being negative below $\sim 5$ at. $\% \mathrm{Cr}$ and positive above. Both $2 \mathrm{BM}$ potentials were fitted to DFT data [12; 44] obtained from special quasi-random structures (SQSs) [45] while CAR was fitted to DFT data [8] obtained from a coherent potential approximation (CPA) [46]. The mixing enthalpy curves from our potential and CAR reproduce the DFT mixing enthalpy very well, both qualitatively and quantitatively. Below $\sim 25$ at. $\% \mathrm{Cr}$ this is also true for OLS; the complete curve, however, takes a symmetric shape, in contrast to the asymmetric DFT shape. As a consequence, the mixing enthalpy takes negative values at the Cr-rich side too, leading to short-range order and high Fe solubility in the Cr-rich phase. These observations contradict DFT data and have never been reported in experimental studies. 
Insert figure 2 around here

The formation energy of intermetallic compounds is another issue to be considered. In Figure 3 the formation energy of 57 intermetallic compounds (as described in [11; 47]) calculated by the three potentials and DFT are compared. In both cases, the configurations were relaxed at constant pressure using a conjugate gradient method. Below 10 at.\% Cr DFT predicts stable intermetallic compounds, consistent with the negative mixing enthalpy for the disordered alloy. Above 10 at.\% Cr, the formation energy of the intermetallic compounds becomes positive and remains so throughout the composition range.

In the Fe-rich region $(<10$ at.\% $\mathrm{Cr})$, the most stable compounds predicted by DFT are also the most stable ones predicted by all three potentials (see Figure 3), with most importantly the lowest compound at 6.67 at.\% Cr. This compound is described by a unit cell with axes oriented along the [210], [1 20] and [001] directions with dimensions $\sqrt{5} a_{0} \times \sqrt{5} a_{0} \times 3 a_{0}$. It contains 30 atoms, of which $2 \mathrm{Cr}$ are separated by $7 \mathrm{nn}$ distance $\left(\sqrt{19} / 2 a_{0}\right)$ along [133]. It should be noted that this compound is consistent with the ordered phase observed in the Monte Carlo simulations by Pareige et al. [48] using OLS. Among the three, both $2 \mathrm{BM}$ potentials also give good quantitative agreement in that concentration range. At higher $\mathrm{Cr}$ composition (> 10 at.\%), OLS generally overstabilizes the compounds thereby even giving negative values at 50 and 93.33 at.\% Cr. The latter is consistent with the low (compared to DFT) and negative mixing enthalpy of the disordered alloy at equiatomic and Cr-rich compositions, respectively. Such negative values in turn may lead to unphysical configurations in the course of e.g. Monte Carlo simulations, and thus constitute a drawback of the potential. The other two potentials do not suffer from the latter problems, although ours still somewhat overstabilizes the compounds.

\section{Insert figure 3 around here}

Experiments have shown the excess vibrational entropy to be non-negligible for the FeCr system [49; 50; 51]; also, it was predicted to have a significant influence on the location of the solubility limit [44]. We calculated the excess vibrational entropy with all three potentials for disordered alloys, based on the harmonic approximation [31] in the $\Gamma$ point applied to cells containing 1024 atoms, prepared as purely random samples.

In Figure 4 excess vibrational entropies for all three potentials are compared with experimental values for the ferro-magnetic phase $[49 ; 51]$. The experimental curves suggest a 
non-negligible contribution to the total excess entropy, amounting to $30-40 \%$ of the configurational entropy for the disordered alloy. As shown in the figure none of the potentials gives a close fit to the experimental values. From the three, however, both 2BM potentials agree best, with ours and OLS closest to the data from [49] and [51], respectively. In the former case the experimental data is underestimated by a factor of about 1.5 ; in the latter it is overestimated (below $\sim 75$ at. $\% \mathrm{Cr}$ ) by about the same factor. The CAR potential, on the other hand, underestimates $S_{\mathrm{xc}}^{\mathrm{vib}}$ by about a factor 3 and gives negative contributions below $\sim 12$ at.\% $\mathrm{Cr}$, which contradicts experiments (see also [44; 52]).

\section{Insert figure 4 around here}

The phase diagrams based on all three potentials are compared to a recently proposed parameterization based on the CALPHAD methodology [53] in Figure 5. The CALPHAD miscibility gap is calculated from an experimentally based [53; 54] Gibbs free energy parameterization. To indicate the ferro to paramagnetic phase transition, the CALPHAD calculated Curie temperature is also added. The phase boundaries for our potential and OLS are obtained from isobaric Monte Carlo simulations in the semi-grand canonical ensemble [55]. In the figure the data points are interpolated by smooth curves to guide the eye. These curves are based on the data points and the fact that full solubility was verified to occur starting from $1200 \mathrm{~K}$ and $800 \mathrm{~K}$ for our potential and OLS, respectively. The phase boundaries for CAR were obtained from free energy calculations using a thermodynamic integration technique (see [52] for more details). Thus in all cases vibrational and configurational entropy were accounted for.

When focussed on the Fe-rich side, we observe large Cr solubility at low temperature, due to the negative heat of mixing. As shown in Figure 5, this behaviour is well reproduced by all potentials, with a particularly close agreement for ours. At about $750 \mathrm{~K}$ the Fe-rich solubility limit increases until full solubility is observed above the critical temperature $\sim 900 \mathrm{~K}$ within the CALPHAD calculation. The curve resulting from OLS underestimates this critical temperature by $\sim 200 \mathrm{~K}$ while our potential overestimates it by roughly the same amount. The critical temperature resulting from CAR, on the other hand, lies above the melting temperature. Note that the difference in critical temperature between OLS and ours closely follows the different trends observed in the excess vibrational entropy. Good agreement of the critical temperature obtained with potentials and CALPHAD shoud not be expected since magnetic interactions are not explicitly included in the former. Below $\sim 25$ at.\%, however, 
direct comparison with the Calphad phase diagram is justified since in this concentration range the Calphad phase boundary is well below the Curie temperature. For higher $\mathrm{Cr}$ concentrations, however, care should be taken as the magnetic transition might significantly affect the solubility, thus close agreement between experiment and potential prediction might not be a concern for potentials that effectively describe the ferro-magnetic phase.

On the Cr-rich side, the Fe solubility approaches zero with decreasing temperature, which is well reproduced by both our potential and CAR. The OLS potential, on the other hand, predicts high Fe-solubility as an unphysical artefact of the negative heat of mixing at the Cr-rich side.

\section{Insert figure 5 around here}

\section{Point-Defect Properties}

The formation, migration and binding energies reported in the current section were calculated in cubic bcc boxes containing 2000 atoms at constant volume $\left(a_{0}=2.8553 \AA\right)$ up to a precision of $1 \mathrm{meV}$. For the formation and binding energies the boxes were relaxed using a conjugate gradient method and the migration energies were estimated using the nudged elastic band method [56].

In Figure 6 the $\mathrm{Cr}-\mathrm{Cr}$ and $\mathrm{Cr}$-vacancy interaction energy $\left(E_{\mathrm{I}}\right)$ in bulk Fe calculated using the three potentials and DFT [12] is presented. The DFT data show $\mathrm{Cr}-\mathrm{Cr}$ repulsion in bulk Fe that decreases fast with increasing distance. This behaviour is mainly responsible for the experimentally observed SRO and is acceptably well reproduced by all potentials. Both 2BM potentials, however, underestimate the difference in repulsion between first and second nearest neighbour $\mathrm{Cr}$ pairs; an effect that is well reproduced by CAR. Concerning the $\mathrm{Cr}$ vacancy interaction, according to both DFT calculations [12] and experiments [57], it is essentially negligible. As shown in Figure 6, this feature is also qualitatively reproduced by all potentials, that predict weak repulsion between $\mathrm{Cr}$ and vacancy. It is important, however, that the interaction energy between $1 \mathrm{nn}$ and $2 \mathrm{nn}$ Cr-vacancy pairs increases, as reproduced by all potentials. This is a prerequisite to obtain the correct order between forward and backward Cr-vacancy exchanges for several chemical environments in the Fe matrix, as discussed further.

\section{Insert figure 6 around here}


In Figure 8 we show the formation energy of interstitial defects in bulk Fe, taking the $\langle 110\rangle$ mixed dumbbell as reference; values predicted by DFT [12] and the three potentials are compared. From the DFT data [12] it follows that the $\langle 110\rangle$ mixed dumbbell configuration in bulk $\mathrm{Fe}$ has the lowest formation energy. However, the inclusion of $\mathrm{Cr}$ in the defect diminishes the difference in formation energy between the $\langle 111\rangle$ and $\langle 110\rangle$ configurations.

In the case of pure $\mathrm{Fe}$, all potentials follow the DFT trend reasonably well, with a proper reproduction of the relative differences between the formation energies of the $\langle 100\rangle$, $\langle 110\rangle$ and $\langle 111\rangle$ configurations being the most important feature. With the introduction of $\mathrm{Cr}$, the data from our potential still captures the shape of the DFT curves reasonably well, while the data from OLS give almost degenerate values for all configurations. The CAR potential, on the other hand, fails to reproduce the $\langle 110\rangle$ mixed dumbbell as the lowest energy configuration.

\section{Insert figure 8 around here}

Next, we compare the binding energy of $\mathrm{Cr}$ interstitials in bulk $\mathrm{Fe}$ obtained from the three potentials with the corresponding DFT values [12]. Such a comparison is presented in Figure 9. As expected from the above results, the CAR potential shows poor agreement with 
DFT data. Given that both 2BM potentials were only fitted to the binding energy between a single $\mathrm{Cr}$ and the $\langle 110\rangle$ dumbbell, they show reasonable agreement with DFT. In particular, our potential is the only one that correctly reproduces the sign for all reported binding energies.

\section{Insert figure 9 around here}

\section{Dislocation-Precipitate Interaction}

Here we address the problem of dislocations in $\mathrm{Fe}$ and $\mathrm{Cr}$ and of the interaction of a $1 / 2\langle 111\rangle$ screw dislocation with $\mathrm{Cr}$ precipitates in the Fe matrix, as obtained from static simulations. The dislocation core energy and radius, shear modulus, Poisson ratio and Peierls stress for a $1 / 2\langle 111\rangle$ screw and a $1 / 2\langle 111\rangle\{110\}$ edge dislocation in the pure metals are summarized in Table 2. Details regarding the calculations can be found in Ref. [59], where the interaction of an edge dislocation with $\mathrm{Cr}$ precipitates was characterized. From Table 2 it appears that the two Fe potentials provide very similar results, whereas the $\mathrm{Cr}$ potentials exhibit at least two essential differences. The shear modulus calculated with our potential is $\sim 30 \%$ smaller, while the Poisson ratio is two times larger than according to OLS. The characteristics of the core of both edge and screw dislocations are similar with both potentials. Finally, we note that the two $\mathrm{Cr}$ potentials predict approximately the same Peierls stress $\left(\tau_{\mathrm{P}}\right)$ for the screw dislocation, which is higher than $\tau_{\mathrm{P}}$ in Fe by $35 \%$ and $55 \%$ according to OLS and our potentials, respectively.

\section{Insert table 2 around here}

The interaction of a screw dislocation with $\mathrm{Cr}$ precipitates was studied in a bcc simulation box with dimensions $20 \times 12 \times 28 \mathrm{~nm}^{3}$ with axes oriented along the [ $\left.\overline{1} \overline{1} 2\right],[1 \overline{1} 0]$ and [111] directions. Periodic boundary conditions were applied in the [111] direction and a straight screw dislocation with left-handed thread and Burgers vector $\boldsymbol{b}=1 / 2[111]$ was created. A Cr precipitate was inserted at a defined distance from the dislocation line and placed so that its centre coincides with the $(1 \overline{1} 0)$ glide plane of the screw dislocation. Then the relaxation was performed to calculate the total energy of the crystal as a function of the distance between the precipitate centre and dislocation line. The precipitate-dislocation interaction energy $E_{\mathrm{I}}$ 
was estimated as $E_{\mathrm{l}}=\left(E^{\mathrm{D}+\mathrm{P}}+E^{\mathrm{Fe}}\right)-\left(E^{\mathrm{D}}-E^{\mathrm{P}}\right)$, following [60] (positive values indicate repulsive interaction). Here $E^{\mathrm{D}+\mathrm{P}}, E^{\mathrm{D}}$ and $E^{\mathrm{P}}$ are the total energy of the crystal containing the dislocation interacting with the precipitate, dislocation only and precipitate only, respectively. $E^{\mathrm{Fe}}$ is the total energy of the Fe crystal with the same geometry and boundary conditions as the crystals used to calculate the above mentioned energies. All crystals were relaxed to reach a convergence of at least $5 \mathrm{meV}$ in the total energy.

As mentioned in section 3 the two $\mathrm{Cr}$ potentials predict different core structures for the screw dislocation in pure Cr. Therefore it is of interest to investigate whether or not the nondegenerate-to-degenerate core structure modification occurs at the precipitate-matrix interface and inside the precipitate. It should be noted that such a modification was found to occur with OLS in our previous study [61]. To address this issue, the core structure of different dislocation segments, selected according to the scheme presented in Table 3, was constructed using the differential displacement (DD) method [42] from the atomic positions obtained after relaxation. The corresponding DD maps are also presented in Table 3. Firstly, we observe that the core structure of the segment located near the centre of the precipitate was found to be the same as in pure $\mathrm{Cr}$ for the corresponding potentials, i.e. the three-fold split core for OLS and isotropic for our potential (Figure 1). The core of the segment penetrating the precipitate normal to its surface (\#1 of Table 3) has a two-fold structure with pronounced split over the precipitate surface, similar for both potentials. However, a difference in the core structure was found for the segments penetrating the precipitate at a tangent to its surface (\#2 and \#3 in Table 3 as the limiting case). With our potential no isotropic or three fold structure modification occurs, contrary to OLS. Additional calculations, not reported here, proved that, with OLS, the isotropic-to-degenerate core transformation occurs not only inside the precipitate but also everywhere near the precipitate matrix interface. The latter implies that in MD simulations such core transformation may manifest itself as emission of kink pairs in the $\{112\}$ plane, thus causing cross-slip movement instead of stable glide (see [43] for details).

\section{Insert table 3 around here}

The interaction energy of the dislocation with precipitates of different sizes as a function of distance between precipitate centre and dislocation line is presented in Figure 10. Qualitatively, the curves look similar for both potentials, showing a maximum in the centre of the precipitate and a fast decrease beyond the precipitate radius. Quantitatively, however, the maximum interaction energy estimated with the OLS is about twice as high considering 
precipitates of the same size. The derivative of the interaction energy with respect to the distance gives an estimate for the interaction force, from which the maximum force (and hence stress) necessary for the dislocation to shear a precipitate can be calculated. Irrespective of the precipitate size and applied potential, the maximum interaction force occurs at $\sim 65 \%$ from the precipitate centre.

\section{Insert figure 10 around here}

The difference in magnitude of the interaction energy between the two potentials can be attributed to the difference in the shear modulus of pure $\mathrm{Cr}$ (see Table 2). For a more correct treatment, however, contributions from the chemical energy (due to additional $\mathrm{Fe}-\mathrm{Cr}$ interface) and dislocation core energy (which differs in $\mathrm{Fe}$ and $\mathrm{Cr}$ ) should not be neglected. Therefore, we have performed additional calculations allowing the total interaction energy to be decomposed into the contributions coming from the shear modulus misfit, chemical and relaxation (related to the strain created around the precipitate) excess energy. The chemical component, $E_{\mathrm{S}}$, was calculated statically, by shearing a precipitate in the $(1 \overline{1} 0)$ plane at its centre by the distance $b$, in a number of steps, each equal to $2 R_{\mathrm{P}} / b$, followed by relaxation. In this way we mimic the shearing of the precipitate due to the advance of the screw dislocation. The shear modulus misfit component, $E_{\mu}$, was calculated by taking the volume integral of the dislocation strain energy between the particle centred at $\left(h_{\mathrm{x}}, h_{\mathrm{y}}, 0\right)$ and a dislocation line parallel to the $\mathrm{z}$ axis, following the expression [62]:

$$
E_{\mu}=\frac{\mu_{P}-\mu_{M}}{\mu_{M}} \int_{P} \rho \cdot D_{Z}(\rho, \theta) \cdot e_{M}(\rho, \theta) \cdot d \rho \cdot d \theta
$$

Here the subscripts $M$ and $P$ indicate respectively matrix and precipitate, $\rho=\sqrt{x^{2}+y^{2}}$ and $\theta=\arctan (x / y)$ are the standard polar coordinates, $e(\rho, \theta)=\mu b^{2} / 8 \pi^{2} \rho^{2}$ is the strain energy density and $D_{Z}$ is expressed as:

$$
D_{Z}(\rho, \theta)=2 \sqrt{R_{P}^{2}-\left(\rho \cos \theta-h_{x}\right)^{2}-\left(\rho \sin \theta-h_{y}\right)^{2}} .
$$

In turn, the excess relaxation energy was calculated as $E_{\mathrm{xc}}=E_{\mathrm{I}^{-}}\left(E_{\mathrm{S}}+E_{\mu}\right)$. 


\section{Summary and Concluding Remarks}

Based on the phase diagram, all potentials are capable of reproducing short-range order and $\alpha^{\prime}$ precipitation. Our potential, however, seems to be the best compromise for thermodynamic modelling. It gives an asymmetric mixing enthalpy, does not stabilize unobserved intermetallic compounds (unlike OLS), provides (though underestimated) a significant amount of excess vibrational entropy (unlike CAR) and a reasonable agreement with the experimental miscibility gap, given that magnetic interactions are not explicitly included in the formalism.

Based on the description of substitutional defect interactions and the phase diagram, both OLS and our potential a priori seem to be the most appropriate choice to simulate thermal annealing in $\mathrm{Fe}-\mathrm{Cr}$ alloys. Besides a proper reproduction of the thermodynamic limit, a reasonable reproduction of vacancy migration barriers, $\mathrm{Cr}-\mathrm{Cr}$ and $\mathrm{Cr}$-vacancy interactions assure that the correct kinetic path is followed using the correct mechanisms. All potentials provide a reasonable description of the $\mathrm{Cr}-\mathrm{Cr}$ and $\mathrm{Cr}$-vacancy interactions, but only the $2 \mathrm{BM}$ potentials provide reasonable values for vacancy migration barriers, with ours following the DFT trends the closest among the two.

Based on the description of interstitial and substitutional defect interactions and the phase diagram, both $2 \mathrm{BM}$ potentials a priori seem to be the most appropriate choice to simulate thermal annealing of $\mathrm{Fe}-\mathrm{Cr}$ alloys under irradiation. Besides a proper description of thermal annealing, a correct stabilization of interstitial complexes is needed to assure that the correct micro-structure is obtained under irradiation. Both 2BM potentials provide reasonable interstitial stabilities (ours in particular), while CAR poorly reproduces interstitial properties. 
Based on the description of the $1 / 2\langle 111\rangle$ screw dislocation core structure (in bulk Fe and $\mathrm{Cr})$ and its stable glide in a $\{110\}$ plane in bulk Fe, our potential a priori seems to be the most suitable choice for the simulation of precipitate-screw dislocation interaction. From the three potentials, ours is the only one reproducing the compact $1 / 2\langle 111\rangle$ screw dislocation core structure in bulk $\mathrm{Cr}$. In addition, our static simulations have revealed an isotropic-todegenerate core transition in every position near the precipitate with OLS that is not seen with our potential. Such a core modification may lead to different interaction mechanisms in dynamic simulations, such as cross slip.

In summary, we have shown that the here developed $\mathrm{Fe}-\mathrm{Cr}$ potential reached the goal of removing the main shortcomings of previously existing ones, while conserving their "good" properties. It should be noted, however, that it is not stiffened to the screened Coulomb interaction and is therefore not suitable to simulate collision cascades in its present form.

\section{Acknowledgements}

The authors thank Drs. D. Nguyen-Manh, M.Yu. Lavrentiev and S. Cottenier for fruitful discussions and their help in preparation of this paper. This research has received partial funding from the European Atomic Energy Community's $7^{\text {th }}$ Framework Programme (FP7/2007-2011), under grant agreement number 212175 (GetMat project). It also contributes to the European Fusion Technology programme (EFDA). The work was partially sponsored by the belgo-argentine MINCYT-FWO bilateral cooperation agreement, Project FW/07/EXII/002. RCP acknowledges support from CONICET-PICT 5062. $\mid$

\section{Appendix A: Tabulation of the Potential Functions}

Tabulations containing up to 5000 points are available on-line as supplementary material related to this paper or at: http://www.ctcms.nist.gov/potentials/ or upon request to the authors. Note that a tabulation of the $s$-embedding functions is not provided due to the singular slope in the origin, so that care should be exercised if used in tabular form. The parameters for the $s$-embedding functions are $A_{1}=-0.217009784, A_{2}=0.388002579$ and $A_{1}=$ $0.00977557632, A_{2}=0.374570104$ for $\mathrm{Fe}$ and $\mathrm{Cr}$, respectively.

\section{Appendix B: Vacancy Migration Barriers}


In this appendix the migration barriers for Fe-Vacancy and Cr-Vacancy exchange for different

local chemical environments are provided. The latter are built by adding $\mathrm{Cr}$ atoms at the six nearest neighbour positions around the migration saddle point. The details are as reported in Table B-1 next.

\section{Insert table B-1 around here}

\section{References}

[1]E.A. Little, Journal of Nuclear Materials 87 (1979) 11.

[2]E.A. Little, D.A. Stow, Metal Science 14 (1980) 89.

[3]S. Porollo, A. Dvoriashin, A. Vorobyev, Y. Konobeev, Journal of Nuclear Materials 256 (1998) 247.

[4]F.A. Garner, M.B. Toloczko, B.H. Sencer, Journal of Nuclear Materials 276 (2000) 123.

[5]Y. Konobeev, A. Dvoriashin, S. Porollo, F. Garner, Journal of Nuclear Materials 355 (2006) 124.

[6]H. Kayano, A. Kimura, M. Narui, Y. Sasaki, K. Suzuki, S. Ohta, Journal of Nuclear Materials 155-157 (1988) 978.

[7]A. Kohyama, A. Hishinuma, D.S. Gelles, R.L. Klueh, W. Dietz, K. Ehrlich, Journal of Nuclear Materials 233 (1996) 138.

[8]P. Olsson, I. Abrikosov, L. Vitos, J. Wallenius, Journal of Nuclear Materials 321 (2003) 84.

[9]A.A. Mirzoev, M.M. Yalalov, D.A. Mirzaev, Physics of Metals and Metallography 97 (2004) 336.

[10]P. Olsson, I.A. Abrikosov, J. Wallenius, Physical Review B 73 (2006) 8.

[11]T.P.C. Klaver, R. Drautz, M.W. Finnis, Physical Review B 74 (2006) 094435.

[12]P. Olsson, C. Domain, J. Wallenius, Physical Review B 75 (2007) 014110.

[13]D. Nguyen-Manh, M.Y. Lavrentiev, S.L. Dudarev, Journal of Computer-Aided Materials Design 14 (2007) 159.

[14]P. Erhart, B. Sadigh, A. Caro, Applied Physics Letters 92 (2008) 3.

[15]I. Mirebeau, M. Hennion, G. Parette, Physical Review Letters 53 (1984) 687.

[16]N.P. Filippova, V.A. Shabashov, A.L. Nikolaev, Physics of Metals and Metallography 90 (2000) 145. 
[17]V.V. Sagaradze, Kositsyna, II, V.L. Arbuzov, V.A. Shabashov, Y.I. Filippov, Physics of Metals and Metallography 92 (2001) 508.

[18]J.O. Andersson, B. Sundman, Calphad-Computer Coupling of Phase Diagrams and Thermochemistry 11 (1987) 83.

[19]G. Bonny, D. Terentyev, L. Malerba, Scripta Materialia 59 (2008) 1193.

[20]P. Olsson, J. Wallenius, C. Domain, K. Nordlund, L. Malerba, Physical Review B 72 (2005) 214119.

[21]A. Caro, D.A. Crowson, M. Caro, Physical Review Letters 95 (2005) 75702.

[22]P. Olsson, J. Wallenius, C. Domain, K. Nordlund, L. Malerba, Physical Review B 74 (2006) 1(E).

[23]G. Bonny, R. Pasianot, L. Malerba, Philosophical Magazine 89 (2009) 711.

[24]M. Mendelev, S. Han, D. Srolovitz, Philosophical Magazine 83 (2003) 3977.

[25]J. Nocedal, S.J. Wright, Numerical Optimization, Springer-Verlag, New York, 2006.

[26]C. Kittel, Introduction to Solid State Physics, John Wiley \& Sons, New York, 1996.

[27]M. Finnis, E. Sinclair, Philosophical Magazine A 50 (1984) 45.

[28]M.S. Duesbery, V. Vitek, Acta Materialia 46 (1998) 1481.

[29]J.H. Rose, J.R. Smith, F. Guinea, J. Ferrante, Physical Review B 29 (1984) 2963.

[30]G.J. Ackland, S.K. Reed, Physical Review B 67 (2003) 174108.

[31]A. Walle van de, G. Ceder, Reviews of Modern Physics 74 (2002) 11.

[32]G. Ackland, M. Mendelev, D. Srolovitz, S. Han, A. Barashev, Journal of Physics: Condensed Matter 16 (2004) 1.

[33]S. Dudarev, P. Derlet, Journal of Physics: Condensed Matter 17 (2005) 1.

[34]L. Malerba, et al., Journal of Nuclear Materials 406(2010) 19.

Deleted: Accepted

[35]C. Domain, G. Monnet, Physical Review Letters 95 (2005) 215506.

[36]J. Wallenius, I. Abrikosov, R. Chakarova, C. Lagerstedt, L. Malerba, P. Olsson, V. Pontikis, B. Sadigh, D. Terentyev, Journal of Nuclear Materials 329-333 (2004) 1175.

[37]H.J. Vanrijn, H.L. Alberts, Journal of Physics F-Metal Physics 13 (1983) 1559.

[38]R. Pasianot, D. Farkas, E.J. Savino, Physical Review B 43 (1991) 6952.

[39]O. Yifang, Z. Bangwei, L. Shuzhi, J. Zhanpeng, Zeitschrift für Physik B 101 (1996) 161.

[40]Z. Bangwei, O. Yifang, L. Shuzhi, J. Zhanpeng, Physical B 262 (1999) 218.

[41]K.W. Katahara, M. Nimalendran, M.H. Manghnani, E.S. Fisher, Journal of Physics FMetal Physics 9 (1979) 2167.

[42]V. Vitek, Crystal Lattice Defects 5 (1974) 1. 
[43]D. Terentyev, G. Bonny, C. Domain, R.C. Pasianot, Physical Review B 81 (2010) 214106.

[44]G. Bonny, R.C. Pasianot, L. Malerba, A. Caro, P. Olsson, M.Y. Lavrentiev, Journal of Nuclear Materials 385 (2009) 268.

[45]A. Zunger, S.H. Wei, L.G. Ferreira, J.E. Bernard, Physical Review Letters 65 (1990) 353.

[46]B.L. Gyorffy, Physical Review B 5 (1972) 2382.

[47]T.P.C. Klaver, G. Bonny, P. Olsson, D. Terentyev, Model. Simul. Mater. Sci. Eng. 18 (2010) 16.

[48]C. Pareige, C. Domain, P. Olsson, Journal of Applied Physics 106 (2009) 104906.

[49]B. Fultz, L. Anthony, J.L. Robertson, R.M. Nicklow, S. Spooner, M. Mostoller, Physical Review B 52 (1995) 3280.

[50]T.L. Swan-Wood, O. Delaire, B. Fultz, Physical Review B 72 (2005) 024305.

[51]M.S. Lucas, M. Kresch, R. Stevens, B. Fultz, Physical Review B 77 (2008) 184303.

[52]G. Bonny, P. Erhart, A. Caro, R.C. Pasianot, L. Malerba, M. Caro, Modelling and Simulation in Materials Science and Engineering 17 (2009) 025006.

[53]G. Bonny, D. Terentyev, L. Malerba, Journal of Phase Equilibrium and Diffusion 31 (2010) 439.

[54]J. Andersson, B. Sundman, Calphad 11 (1987) 83.

[55]G. Bonny, R.C. Pasianot, E. Zhurkin, M. Hou, in preparation (2010).

[56]G. Henkelman, H. Jonsson, Journal of chemical physics 113 (2000) 9978.

[57]A. Moslang, H. Graf, G. Balzer, E. Recknagel, A. Weidinger, T. Wichert, R.I. Grynszpan, Physical Review B 27 (1983) 2674.

[58]D. Nguyen-Manh, M.Y. Lavrentiev, S.L. Dudarev, Comptes Rendus Physique 9 (2008) 379.

[59]D. Terentyev, G. Bonny, L. Malerba, Acta Materialia 56 (2008) 3229.

[60]T. Harry, D.J. Bacon, Acta Materialia 50 (2002) 209.

[61]D. Terentyev, G. Bonny, L. Malerba, Nuclear Instruments \& Methods in Physics Research Section B-Beam Interactions with Materials and Atoms 267 (2009) 3155.

[62]E. Nembach, Physica Status Solidi A-Applied Research 78 (1983) 571.

[63]W. Pearson, A Handbook of Lattice Spacings and Structures of Metals and Alloys, Pergamon Press, New York, 1958.

[64]G. Loper, L. Smedskjaer, M. Chason, R. Siegel, Positron Annihilation. in: P. Jain, (Ed.), World Scientific, Singapore, 1985, pp. 461. 
[65]H. Schultz, Materials Science and Engineering A-Structural Materials Properties Microstructure and Processing 141 (1991) 149.

[66]S. Cottenier, Private communication (2009).

[67]Y.N. Osetsky, D.J. Bacon, Modelling and Simulation in Materials Science and Engineering 11 (2003) 427.

[68]D. Terentyev, D. Bacon, Y. Osetsky, Journal of Physics: Condensed Matter 20 (2008) $\underline{445007 .}$.

\section{Table and Figure Captions}

Table 1 - Summary of the basic properties of both Cr potentials.

Table 2 - Properties of $\mathrm{Fe}$ and $\mathrm{Cr}$ as obtained from the potentials.

Table 3 - Differential displacement maps drawn in the (111) zone, for a screw dislocation interacting with a $2 \mathrm{~nm}$ precipitate. The thick dashed line shows the edge of the precipitate and a double arrow shows the directions of the preferential split of the dislocation core. The centre of the dislocation core is shown by symbol $\S$.

Table B-1 - The vacancy migration barrier for different local Cr configurations, with the first and second value denoting the forward and backward jump, respectively. In the figure black circles are $\mathrm{Fe}$, white ones are $\mathrm{Cr}$, in grey is the migrating atom, and the square represents the vacancy.

Figure 1 - Comparison of the screw component of differential displacement maps obtained with our potential (a) and the O05 potential (b).

Figure 2 - Comparison of the mixing enthalpies obtained from the potentials with DFT data $[8 ; 12 ; 44]$.

Figure 3 - Comparison of the formation energy obtained by the different potentials with the corresponding DFT values $[11 ; 47 ; 48]$ for various intermetallic compounds.

Figure 4 - Comparison of the excess vibrational entropy obtained from the different potentials with the experimental ones from $[49 ; 51]$.

Figure 5 - Comparison of the phase diagrams resulting from the different potentials against a CALPHAD based calculation. The curves for the OLS and our potential were taken from [55], while for CAR and CALPHAD they were taken from [52] and [53], respectively. Figure 6 - Comparison between DFT [12] and all three potentials of the interaction energy between $\mathrm{Cr}-\mathrm{Cr}$ and $\mathrm{Cr}$-vacancy pairs in bulk $\mathrm{Fe}$. 
Figure 7 - Comparison between DFT [58] and the three potentials of the average vacancy migration barrier in bulk Fe for different local $\mathrm{Cr}$ configurations around the saddle.

Figure 8 - Formation energies of different interstitial configurations in bulk $\mathrm{Fe}$ and $\mathrm{Cr}$, calculated using DFT [12] and the three different potentials.

Figure 9 - Comparison between DFT [12] and the three potentials of the binding energy of $\mathrm{Cr}$ interstitial configurations.

Figure 10 - Precipitate dislocation interaction energy as function of the distance for our (left) and the OLS potential (right). The position of maximal force between precipitate and dislocation is indicated by $F_{\mathrm{MAX}}$.

Figure 11 - Different contributions to the interaction energy (top row) and interaction force (bottom row) for our (left) and the OLS potential (right), for a $4 \mathrm{~nm} \mathrm{Cr}$ precipitate. The position of the precipitate edge and maximal force between precipitate and dislocation are indicated by $R_{\mathrm{P}}$ and $F_{\mathrm{MAX}}$, respectively.

\section{Tables}

Table 1

\begin{tabular}{lccc}
\hline & Experiment or DFT & This Work & O05 \\
\hline$a_{0}(\AA)$ & $2.878^{\mathrm{a}} / 2.834^{\mathrm{b}}$ & 2.866 & 2.878 \\
$B(\mathrm{GPa})$ & $208^{\mathrm{c}}$ & 215 & 208 \\
$C^{\prime}(\mathrm{GPa})$ & $152^{\mathrm{c}}$ & 155 & 152 \\
$C_{44}(\mathrm{GPa})$ & $105^{\mathrm{c}}$ & 108 & 105 \\
$E_{\mathrm{coh}}(\mathrm{eV})$ & 4.10 & 4.10 & 3.84 \\
$E_{\mathrm{f}}^{\mathrm{V}}(\mathrm{eV})$ & $2.0 \pm 0.2^{\mathrm{d}} / 2.59^{\mathrm{b}}$ & 2.52 & 2.56 \\
$E_{\mathrm{m}}^{\mathrm{V}}(\mathrm{eV})$ & $0.95^{\mathrm{e}}$ & 0.94 & 0.99 \\
$E_{\mathrm{coh}}^{\mathrm{fcc}}-E_{\mathrm{coh}}^{\mathrm{bcc}}(\mathrm{eV})$ & $-0.4^{\mathrm{f}}$ & -0.16 & -0.03 \\
$E_{\mathrm{f}}^{<110>}-E_{\mathrm{f}}^{<111>}(\mathrm{eV})$ & $-0.02^{\mathrm{b}}$ & -0.07 & -0.02 \\
$E_{\mathrm{f}}^{<110>}-E_{\mathrm{f}}^{<100>}(\mathrm{eV})$ & $-1.12^{\mathrm{b}}$ & -0.58 & -1.23
\end{tabular}

${ }^{\mathrm{a}}$ Experiment, Ref. [63]

${ }^{\mathrm{b}}$ DFT, Ref [20]

${ }^{\mathrm{c}}$ Experiment, Ref. [41]

${ }^{\mathrm{d}}$ Experiment, Ref. [64]

${ }^{\mathrm{e}}$ Experiment, Ref. [65] 
${ }^{\mathrm{f}}$ DFT, Ref. [66]

Table 2

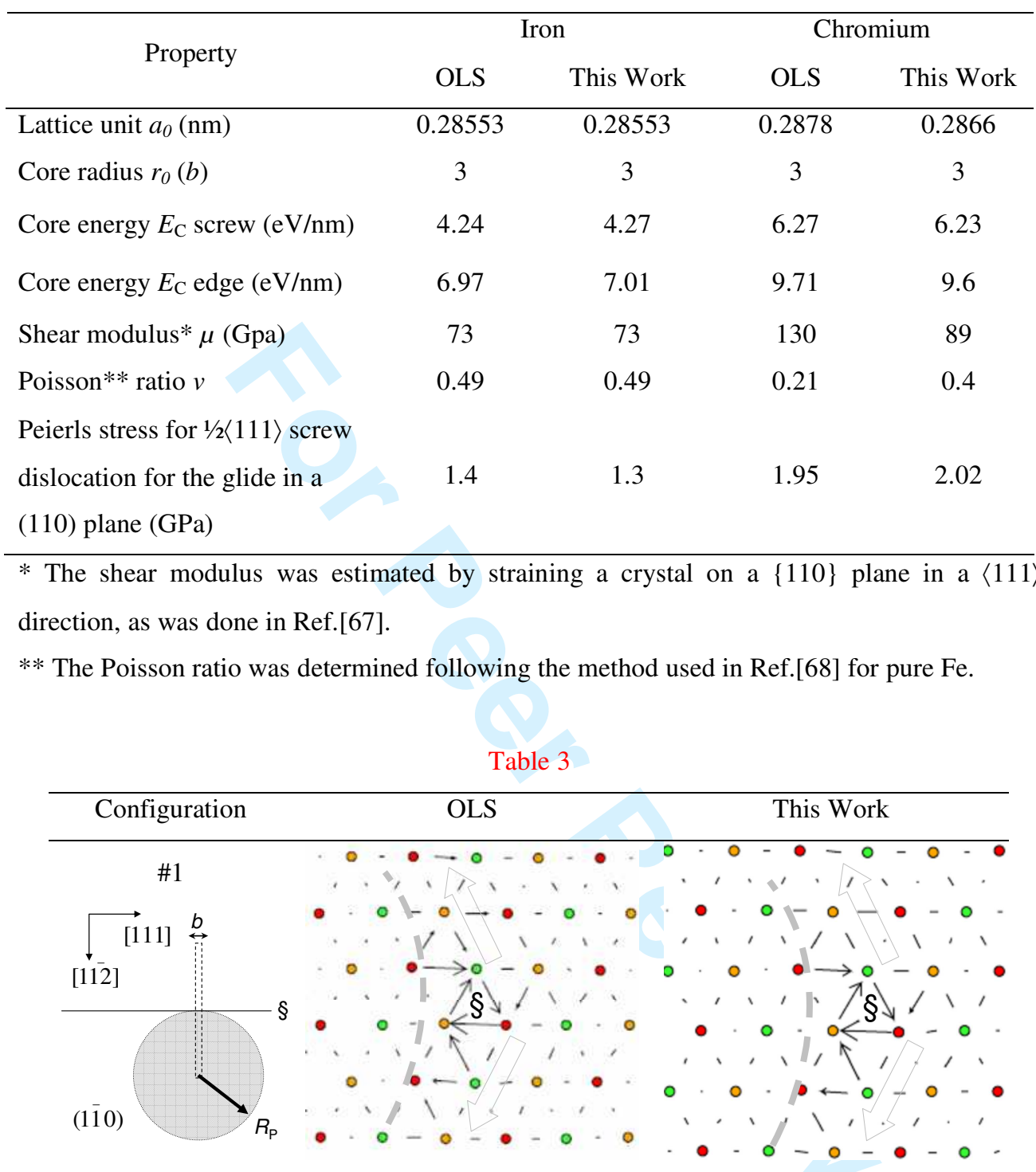




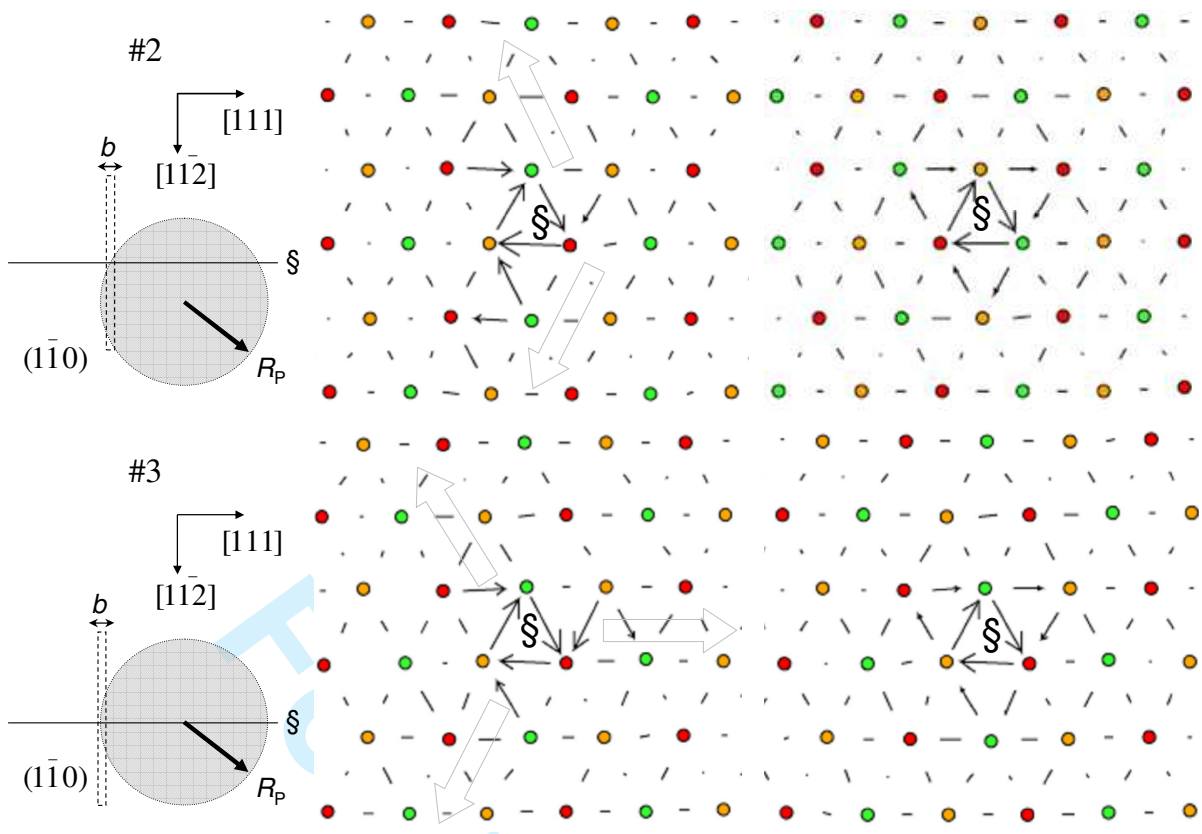

Table A-1

\begin{tabular}{|c|c|c|c|c|c|}
\hline Configuration & & DFT & This Work & OLS & CAR \\
\hline & $E_{\mathrm{m}}^{\mathrm{Cr}}$ & $0.57 / 0.57$ & $0.57 / 0.57$ & $0.56 / 0.56$ & $0.90 / 0.90$ \\
\hline & $E_{\mathrm{m}}^{\mathrm{Fe}}$ & $0.64 / 0.64$ & $0.63 / 0.63$ & $0.64 / 0.64$ & $0.63 / 0.63$ \\
\hline & $E_{\mathrm{m}}^{\mathrm{Cr}}$ & $0.72 / 0.54$ & $0.63 / 0.57$ & $0.61 / 0.53$ & $0.97 / 0.87$ \\
\hline & $E_{\mathrm{m}}^{\mathrm{Fe}}$ & $0.66 / 0.60$ & $0.65 / 0.63$ & $0.67 / 0.63$ & $0.64 / 0.62$ \\
\hline & $E_{\mathrm{m}}^{\mathrm{Cr}}$ & $0.79 / 0.52$ & $0.66 / 0.58$ & $0.62 / 0.51$ & $1.00 / 0.85$ \\
\hline & $E_{\mathrm{m}}^{\mathrm{Fe}}$ & $0.73 / 0.57$ & $0.67 / 0.63$ & $0.68 / 0.61$ & $0.65 / 0.61$ \\
\hline & $E_{\mathrm{m}}^{\mathrm{Cr}}$ & 1.09 / 0.69 & $0.68 / 0.58$ & $0.59 / 0.49$ & $0.99 / 0.82$ \\
\hline & $E_{\mathrm{m}}^{\mathrm{Fe}}$ & $0.84 / 0.55$ & $0.69 / 0.63$ & $0.67 / 0.60$ & $0.65 / 0.59$ \\
\hline & $E_{\mathrm{m}}^{\mathrm{Cr}}$ & $0.88 / 0.55$ & $0.67 / 0.61$ & $0.56 / 0.51$ & $0.86 / 0.77$ \\
\hline & $E_{\mathrm{m}}^{\mathrm{Fe}}$ & $0.78 / 0.58$ & $0.68 / 0.64$ & $0.66 / 0.62$ & $0.57 / 0.55$ \\
\hline
\end{tabular}




\begin{tabular}{cccccc}
\hline $\mathrm{Cr}$ & $0.74 / 0.56$ & $0.66 / 0.62$ & $0.54 / 0.52$ & $0.76 / 0.72$ \\
\hline & $E_{\mathrm{m}}^{\mathrm{Cr}}$ & $0.70 / 0.61$ & $0.67 / 0.65$ & $0.65 / 0.64$ & $0.50 / 0.50$ \\
\hline & $E_{\mathrm{m}}^{\mathrm{Fe}}$ & $0.64 / 0.64$ & $0.66 / 0.66$ & $0.64 / 0.64$ & $0.47 / 0.47$ \\
\hline
\end{tabular}

Figures
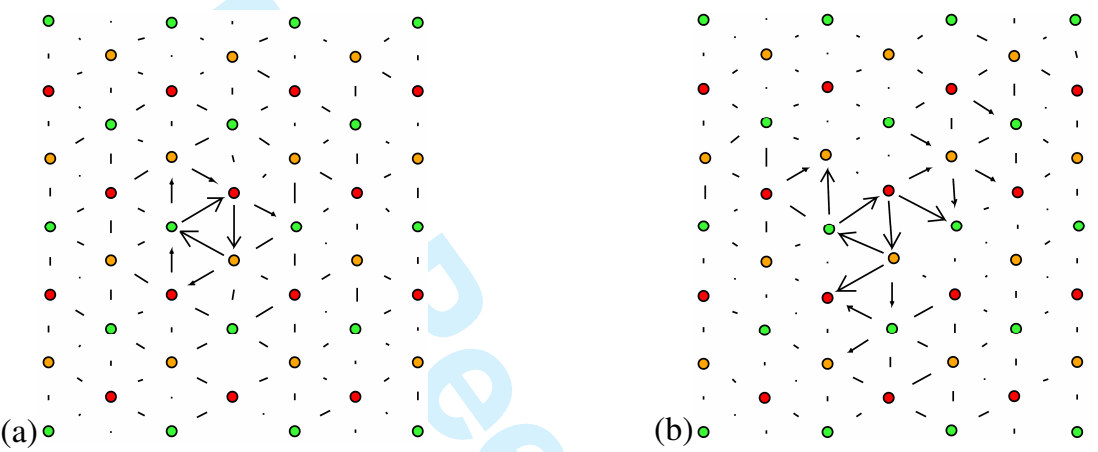

Figure 1
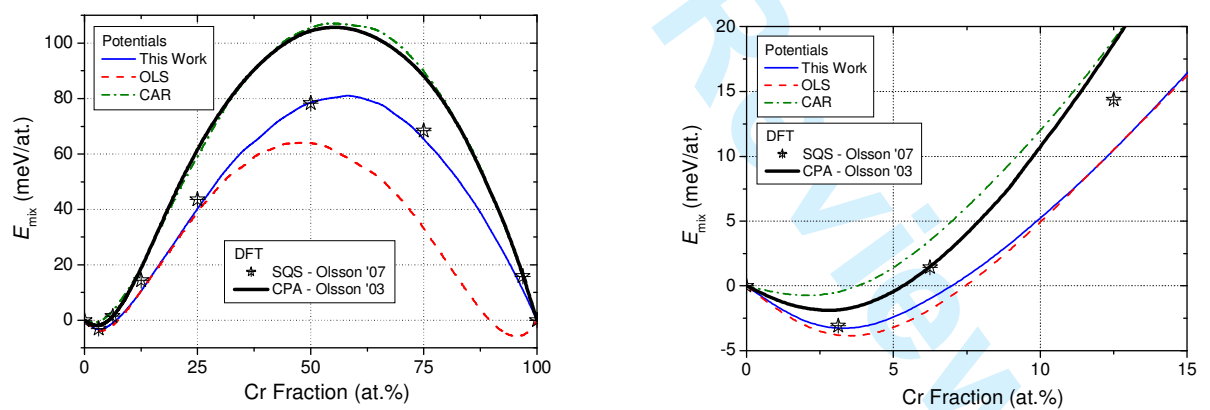

Figure 2 

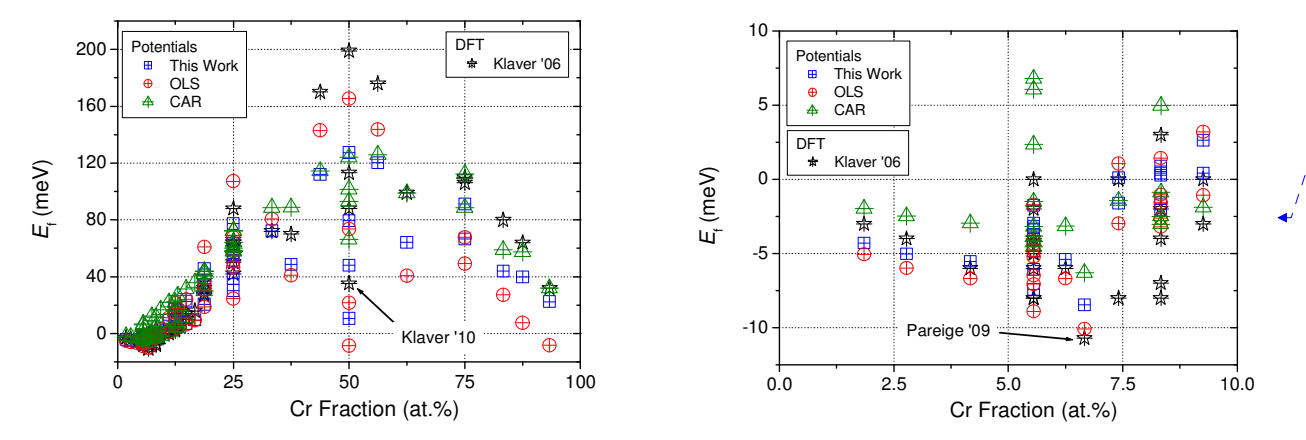

Figure 3

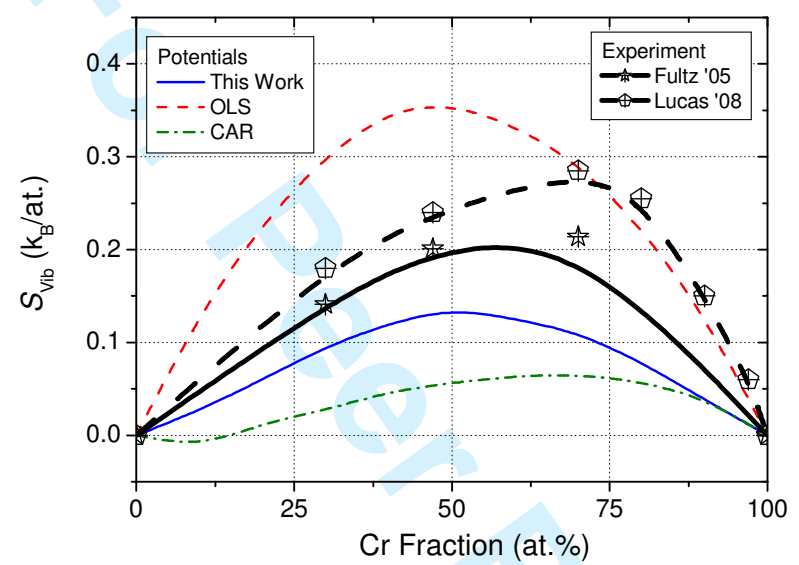

Figure 4 


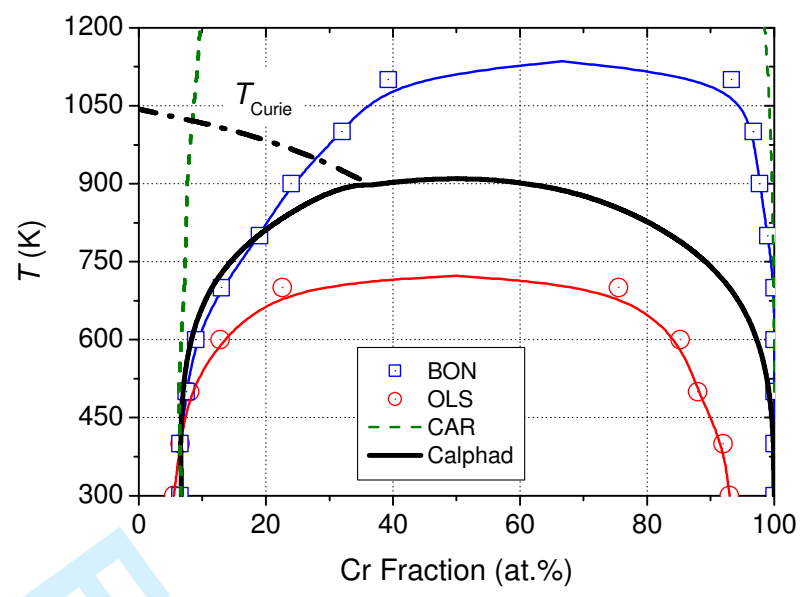

Figure 5

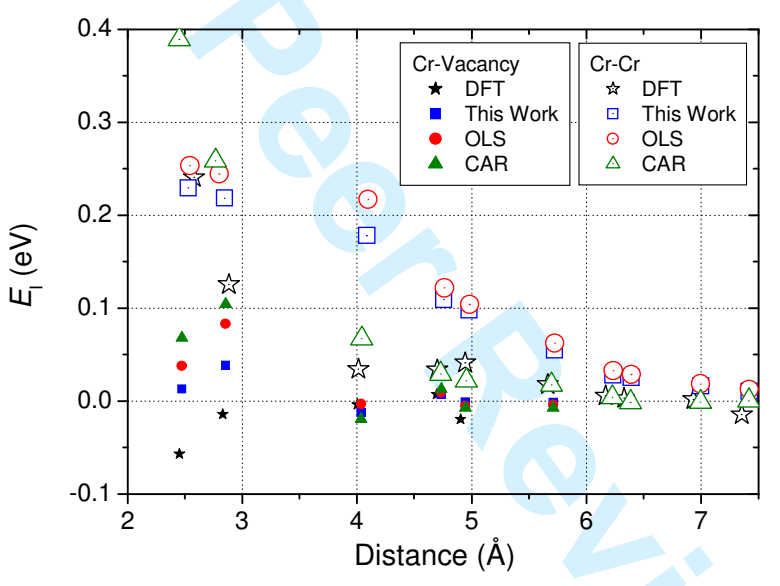

Figure 6 

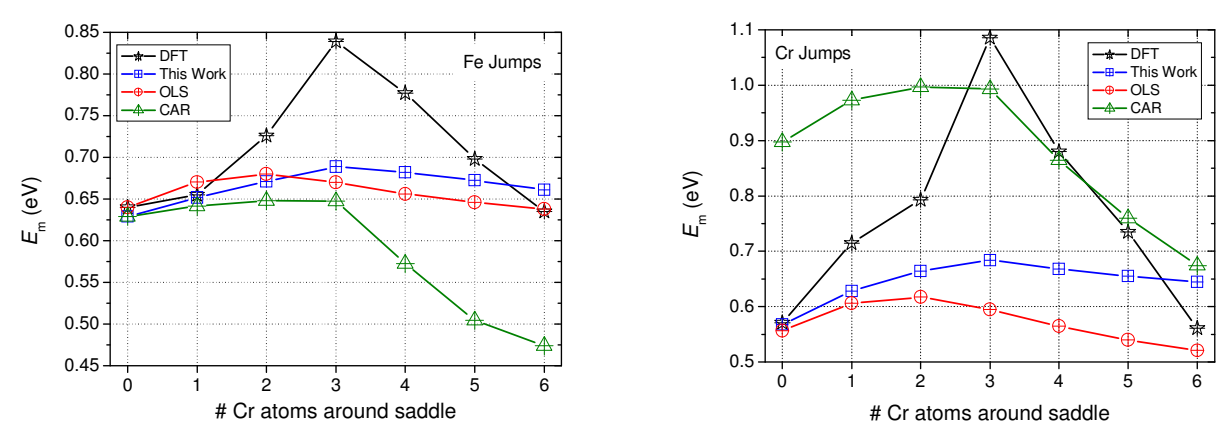

Figure 7
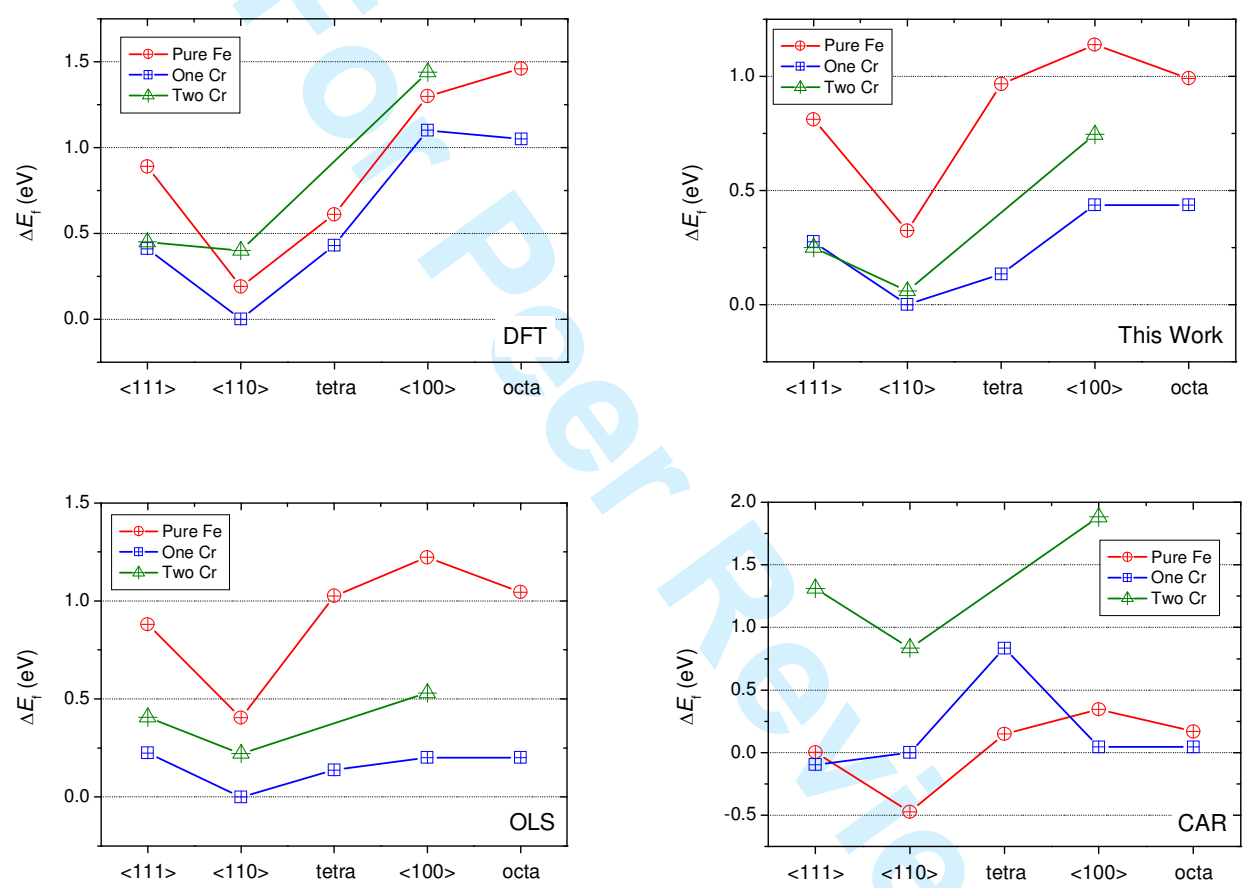

Figure 8 


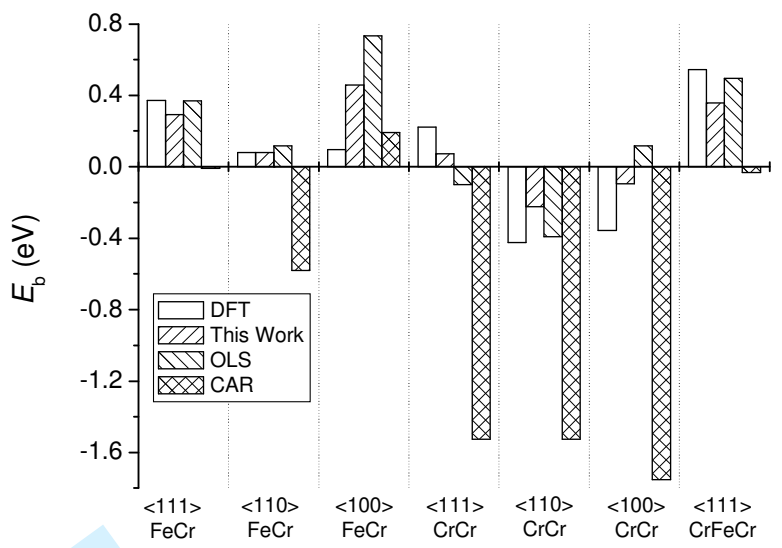

Figure 9
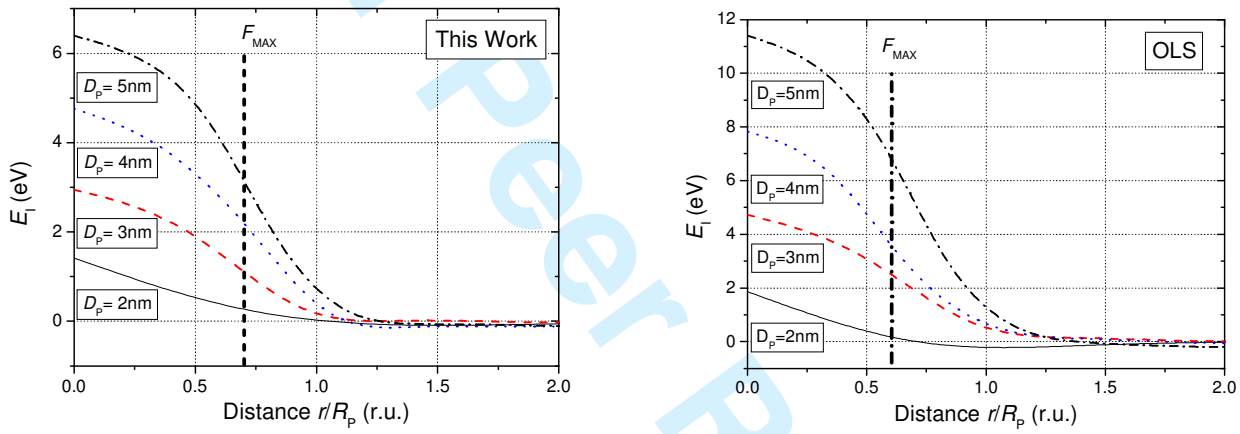

Figure 10
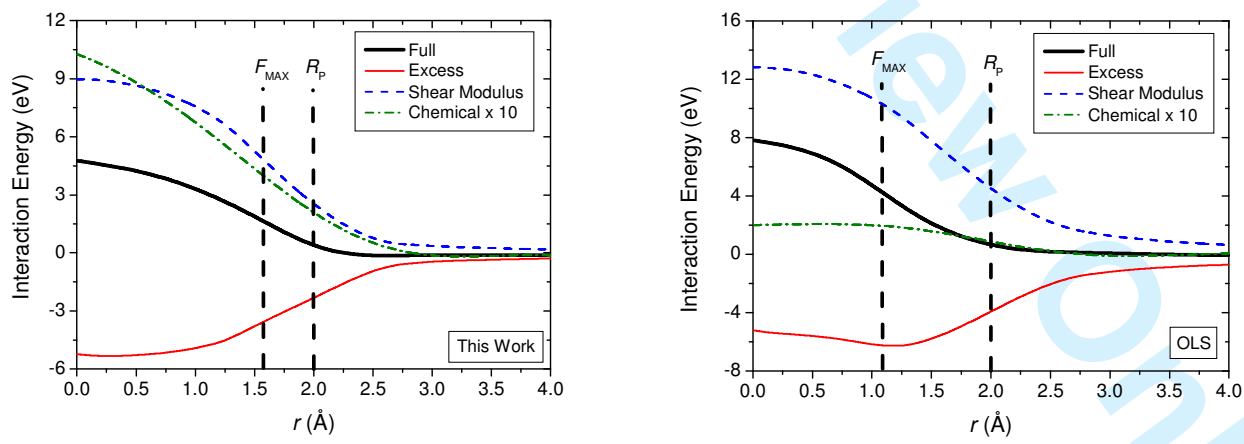

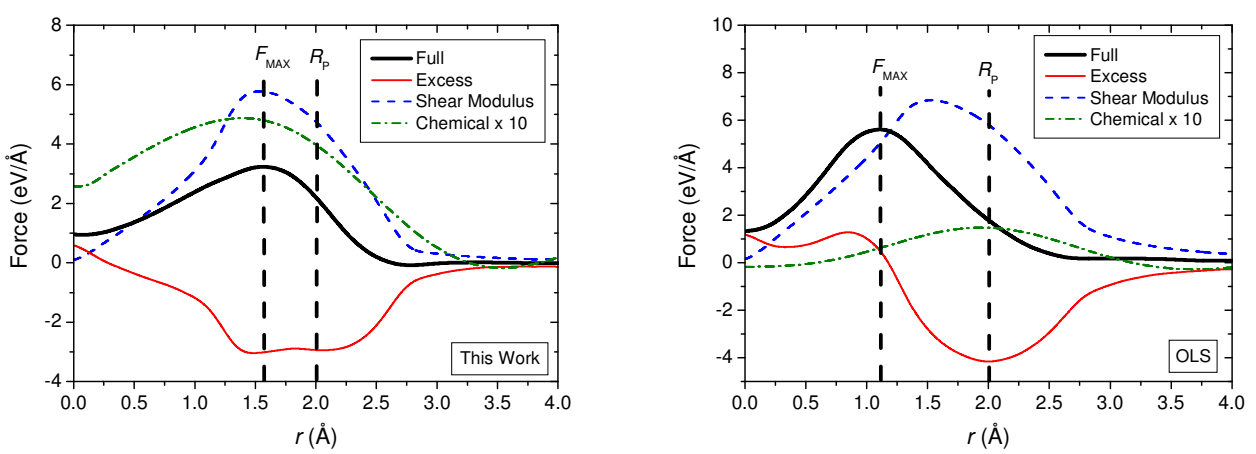

Figure 11 